\title{
Procedural memory in infancy: Evidence from implicit sequence learning in an eye-tracking paradigm
}

Felix-Sebastian Koch, Anett Sundqvist, Ulrika Birberg Thornberg, Sandra Nyberg, Jarrad A. G. Lum, Michael T. Ullman, Rachel Barr, Mary Rudner and Mikael Heimann

The self-archived postprint version of this journal article is available at Linköping University Institutional Repository (DiVA):

http://urn.kb.se/resolve?urn=urn:nbn:se:liu:diva-164656

N.B.: When citing this work, cite the original publication.

Koch, F., Sundqvist, A., Birberg Thornberg, U., Nyberg, S., Lum, J. A. G., Ullman, M. T., Barr, R., Rudner, M., Heimann, M., (2020), Procedural memory in infancy: Evidence from implicit sequence learning in an eye-tracking paradigm, Journal of experimental child psychology (Print), 191, 104733. https://doi.org/10.1016/j.jecp.2019.104733

Original publication available at:

https://doi.org/10.1016/j.jecp.2019.104733

Copyright: Elsevier

http://www.elsevier.com/

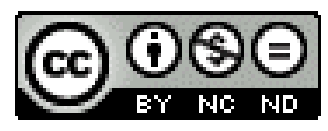




\title{
Procedural memory in infancy:
}

\section{Evidence from implicit sequence learning in an eye-tracking paradigm}

Running title: Procedural memory in infancy

\author{
Felix-Sebastian Koch \\ Linköping University, Sweden \\ Annette Sundqvist \\ Linköping University, Sweden \\ Ulrika Birberg Thornberg \\ Linköping University, Sweden \\ Sandra Nyberg \\ Linköping University, Sweden \\ Jarrad A.G. Lum \\ Deakin University, Australia \\ Michael T. Ullman \\ Georgetown University, USA \\ Rachel Barr \\ Georgetown University, USA \\ Mary Rudner \\ Linköping University, Sweden \\ Mikael Heimann \\ Linköping University, Sweden
}

Word count: 9919 words 
Author Note

Felix-Sebastian Koch, Infant and Child Lab, Department of Behavioural Sciences and Learning, Linköping University, Sweden;

Correspondence concerning this article should be addressed to Felix-Sebastian Koch, Infant and Child Lab Linköping, Department of Behavioural Sciences and Learning, Linköping University, SE-581 83 Linköping, Sweden. E-mail: felix.koch@liu.se

Conflict of interest statements: We have no conflict of interest to disclose.

Acknowledgements: This research was supported by a grant from the Swedish Research Council (Vetenskapsrådet) to Mikael Heimann (grant 2016-01033). We wish to thank the families and adult participants that participated in this research.

(C) 2019. This manuscript version is made available under the CC-BY-NC-ND 4.0 license http://creativecommons.org/licenses/by-nc-nd/4.0/ 


\section{Procedural memory in infancy: Evidence from implicit sequence learning in an eye-tracking paradigm}

Multiple systems appear to underlie learning and memory abilities (Eichenbaum, 2010; Schacter, Wagner, \& Buckner, 2000; Squire, 2004). However, the development of procedural memory, the memory system that underlies the learning of habits and skills, has not been fully tracked from infancy to adulthood. Procedural memory involves the acquisition of particular motor or cognitive skills or habits, and is implicated in the skills and habits that underlie language learning (Ullman, 2016) and social interaction (Lieberman, 2000). Skills and habits emerge in everyday life as the repeated execution of a task leads to improvements in the speed and/or accuracy with which the task is completed (Magallón, Narbona, \& Crespo-Eguílaz, 2016; Squire, 2009; Zwart, Vissers, Kessels \& Maes 2017). Using the same measure for infants and adults has important benefits for tracking the development of procedural memory as performance can be directly compared. For example, different looking paradigms have been used to test infants and adults. The visual paired comparison test is a looking paradigm, now understood to tap declarative memory (Manns,

Stark \& Squire, 2000), which is well established as a measure in infancy that can also be used with adults (Richmond, Sowerby, Colombo, \& Hayne, 2004). Statistical learning has also been examined in infants, children, and adults using a looking paradigm (Amso \& Davidow, 2012), and the authors suggested that statistical learning showed developmental invariance based on observation of few or no differences in response patterns between age groups. However, procedural memory is frequently studied by investigating changes in motor behavior during sequence learning, and as such has not been tracked from infancy to adulthood.

The Serial Reaction Time (SRT) task and related tasks which measure sequence learning have frequently been used to examine the development of procedural memory 
over the life-span beginning at age 4 years (Adi-Japha, Badir, Dorfberger, \& Karni, 2014;

Howard, Howard, Dennis, \& Kelly, 2008; Janacsek, Fiser, \& Nemeth, 2012; Zwart et al., 2017).

Nissen and Bullemer (1987) developed the original SRT procedure to investigate sequence learning. Participants pressed one of four keys in response to corresponding locations where a visual cue could appear on a screen. The spatiotemporal order was either random or determined by repeating a 10-item sequence 10 times in each block for eight blocks in total, without indication of when the sequence or the blocks started or ended. In the random condition, participants performed the same number of trials and blocks. Results indicated that participants in the sequence condition improved in performance, with mean reaction time decreasing over blocks, whereas participants in the random condition did not. Even though the original study lacked a within participant comparison, this SRT procedure became very influential in testing procedural memory. Recent studies (e.g. Karatekin, Marcus, \& White, 2007; Lum, Kidd, Davis, \& Conti-Ramsden, 2010) have added one or more random blocks after repeating blocks to confirm within-participant effects. When a random block is introduced, reaction time typically slows. This is because the predictions the participant has learned based on the repeated sequence are no longer valid. If a final sequence block is reintroduced, participants once again exhibit the learned behavior and reaction time decreases (Karatekin et al., 2007). This systematic decrease in reaction time during sequence blocks and increase in reaction time during random block trials is the hallmark of the SRT task (Robertson, 2007). The final sequence block is a manipulation check to ensure that any observed response time increase between the sequence block prior to the random block and the random block itself was not simply due to fatigue.

The kind of sequence that is used also varies among studies. In first order sequences, such as those used by Nissen and Bullemer (1987) and Lum et al. (2010), the 
spatiotemporal order of visual stimuli can be predicted from the preceding item, with varying degrees of probability. Second order sequences, such as used by Karatekin et al. (2007) are also common. In these sequences, the location of an upcoming visual stimulus can be predicted by combining the two preceding items, but the preceding item is not predictive in itself. Howard and Howard (1997) introduced the alternating SRT task (ASRT task), another frequently used variation (e.g. Howard \& Howard, 2013; Nemeth, Janacsek, \& Fiser, 2013) of the target sequence that focuses on second order sequences. In the ASRT task, the position of every second appearance of the visual stimulus follows a given spatiotemporal sequence, whereas locations of intermittent items are chosen randomly. The development of procedural memory might be invariant from childhood to adulthood. For example, Karatekin et al. (2007) examined four cross-sectional age groups from 8-year-olds to young adults. Further, Lum et al. (2010) also found little evidence of change in performance in a longitudinal study from age 5.5 years to age 6.5 years. However, some studies found evidence for change in procedural memory. Examining sequence learning over the life span from age 4 years, Janacsek and colleagues (2012) found evidence for sequence learning to be most efficient until age 11 years with a decline in performance from that age onwards. Lukács and Kemény (2015) also found evidence for change and argued for an inverted U-shaped pattern of performance on the SRT task across the life span with a peak at age 30 years. However, data analysis method seems to influence the nature of the change observed in procedural memory. Janacsek and colleagues (2012) pointed out that if their data were corrected for baseline performance by normalizing the reaction times of the participants, the data indicated that children increase their efficiency for sequence learning between 4 and 11 years of age. Zwart and colleagues (2017) conclude in their metaanalysis that studies that found increases in sequence learning ability from school-age to 
young adulthood are consistent if data were normalized. But if one considers raw data, children are consistent in their ability to learn from school-age onwards. This pattern of results underscores the need for a single task that can be used over the life-span starting from infancy. In the current study, normalized data will be reported, as normalization of the data in some studies indicated an increase in the sequence learning ability during childhood. If the ability to learn sequences increases during childhood, showing effects of sequence learning ability in infants for normalized data would be a more robust test of sequence learning in infancy, as effects would be smaller in normalized data.

Due to the lack of appropriate measures applicable across the lifespan, the developmental trajectory of procedural memory has not been fully documented from infancy to adulthood. A key problem in designing studies to examine procedural learning and memory in infants is connected with infants' slowly developing control of the motor actions often involved in tests of procedural learning. This has excluded them from most procedural tasks that involve motor control, including the SRT (Nissen \& Bullemer, 1987) and related tasks. One way to circumvent the infant motor control problem is to instead utilize the oculomotor system, which matures quickly during the first year of life (Braddick \& Atkinson, 2011; Scerif et al., 2005). Vakil, Bloch, and Cohen (2017) compared reaction times based on manual responses to oculomotor responses and found similar sequence learning in both measures in a study with adults. In the present study, we therefore capitalized on infants' mature eye movements by developing an infant-friendly ocular-motor adaptation of the SRT task that can be used to examine procedural memory in both infants and adults. Instead of button presses, saccades, that is, rapid eye movement from one visually fixated location to another, are measured. 
Previous work based on the timing of saccades suggests that infants can learn visual sequences. Using the visual expectation paradigm (VExP), Haith and colleagues

demonstrated that infants learn to expect the next item to appear in a particular location and can make an eye movement in anticipation of the item appearing there (Canfield \& Haith, 1991; Canfield, Smith, Brezsnynak, \& Snow 1997; Haith, Hazan, \& Goodman, 1988). In this procedure, visual stimuli are presented in the left or right visual field followed by a one second interval when no stimulus is presented. During this interval, infants perform anticipatory saccades to the anticipated visuospatial location of the upcoming stimulus, indicating expectation-based learning of the visuospatial sequence (e.g. Canfield \& Haith, 1991). Note that although infants do perform anticipatory saccades significantly more often to upcoming locations of stimuli, most saccades actually occur after the item appears. However, these reactive saccades are also taken to index learning, since even reactive saccades to a predictable sequence item location are executed earlier than baseline (Canfield \& Haith, 1991, Haith et al., 1988). The complexity of the visual sequences that can be learned as measured with the VExP increases developmentally. Canfield and Haith (1991) reported that 2-month-olds could learn an alternating left-right pattern, while 3-month-olds learned more complex asymmetric left-left-right patterns. Six to 12-month-olds learned an even more complex visuospatial sequence, with anticipatory saccades increasing and reaction time decreasing from 6 to 9 months but no additional changes occurring between 9 and 12 months (Reznick, Chawarska, \& Betts, 2000). The VExP differs from the SRT procedure since the VEXP paradigm lacks a comparison between a learned sequence and a random sequence. Instead VExP measures saccades toward the correct (upcoming) position compared to saccades to the wrong position. 

old infants watched objects appear one at a time in one of six different locations in a $2 \times 3$ grid. The location of each item was either unpredictable or perfectly predictable based on the location of the previous item. The study revealed that the infants looked longer at stimuli with novel and unpredictable sequences compared to predictable sequences that included the learned spatiotemporal order, indicating sequence learning. Although these findings indicate a capacity for learning visual-spatial sequences, analogous to findings from SRT, it is difficult to directly compare performance on these infant tasks with data collected with older children and adults on SRT tasks. It is for this reason that we developed an ocularmotor SRT task for infants.

The current study protocol used a first order sequence (Lum et al., 2010; Nissen \& learning has been linked to activity of the basal ganglia and thus implicated in procedural memory processes in adults (Clark, Lum, \& Ullman 2014). Procedural memory, as opposed to declarative memory, is usually defined as inaccessible to conscious recollection (Squire, 2009) and scientific attention has been aimed at designing studies where participants are unaware of the learned sequences (Song, Howard, \& Howard, 2007; Stark-Inbar, Raza, Taylor, \& Ivry 2017). For example, to reduce awareness, Song and colleagues embedded the repeating sequence within an alternating pattern, using the alternating SRT. Awareness of a learned sequence might affect how flexibly the learned representation can be used (Esser \& Haider, 2017) and might increase variability in performance (Yordanova, Kirov, \& Kolev, 2015). Structural features of the target sequence (e.g. its length) influence how easy it is for participants to become aware of the target sequence and perceptually salient features of 
the stimuli can emphasize but also distract from the structural features of the target

145

sequence. Thus, in the current study we aimed to strike a balance between a sequence that infants are able to learn and that adults cannot verbally describe. One adaptation introduced in the current study is the use of infant friendly images, a novel one for each trial, instead of using the same visual stimulus, an asterisk or a dot, throughout (Robertson, 2007). This changes the perceptually salient features of the stimuli used and adds the possibility of verbal processing for each image, but the spatiotemporal structure is unchanged. It is important to note that although the spatial pattern repeats, the images change across trials, meaning that it is more difficult to detect the repeating spatial sequence and that even if items are labelled by participants, verbal processing will not aid sequence learning. Changes in eye movements towards predictable stimuli have also been used to index procedural learning in adults. Specifically, saccade latency has been used to measure procedural learning on the SRT task with adult participants (Marcus, Karatekin, \& Markiewicz, 2006; Vakil el al., 2017) and children aged 8 years and older (Karatekin et al., 2007). Saccade latency is comparable to motor response time. Instead of measuring time taken to press the button, reaction time is measured from the appearance of a stimulus at a new location until the participant starts to saccade to the new location. This indexes how quickly a person shifts her attention to a new visuospatial location. The assumption is the same as with manual SRT tasks; that is, a reaction to a predictable location should be faster than a reaction to a random location. Thus, both manual and oculomotor reaction times tap procedural memory (Marcus et al., 2006; Vakil et al., 2017). The SRT has been widely used in older children with productive language and adults (Lum, Ullman, \& Conti-Ramsden, 2013; Magallón et al., 2016; Robertson, 2007) but to the best of our knowledge not during infancy. 
In the present study, we tested a new ocular-motor adaptation of the SRT task that

is suitable for infants, but that could be used with adults as well. Participants were presented with 125 trials divided into 5 blocks. Each trial presented a novel infant-friendly image in one of three locations on a screen, with the new location always being different to the previous trial's location. In the first three blocks and the final block the spatiotemporal order of items was defined by a five-item spatiotemporal sequence. In the fourth block the spatiotemporal order was random.

The critical index of SRT sequence learning in this ocular-motor adaptation of the SRT task, as in SRT tasks in general, is significantly longer mean reaction time in the random block compared to both the immediately preceding and immediately following recurring sequence blocks (Robertson, 2007). Therefore, we examine mean reaction times within these three blocks and predict that saccade latencies would be significantly longer in the random block compared to the preceding as well as the following sequence block, for all participants, infants and adults alike. Further, in order for the disruption in the critical phase to be plausibly linked to procedural memory some kind of learning would need to occur during a learning phase, which in the current study is represented by the first three blocks. A significant increase in reaction time across the learning phase would invalidate the ocularmotor SRT task. However, we expect the participants to demonstrate a decrease in mean saccade reaction times across the learning phase. Given prior studies of infant learning (for review see Rovee-Collier \& Barr, 2010), we predicted that mean reaction times would decrease more in infants than adults. In addition, in order to check whether the adults had any explicit knowledge of the recurring sequences after taking the ocular-motor SRT task we asked them to report any sequences that they noted.

\section{Method}


Participants. The present study was approved by the Regional Ethical Review Board,

192

193

Linköping, Sweden (2017/386-32).

Infants. Parents were invited by mail to participate with their infants in developmental psychology research. Those who expressed an interest were contacted by phone and informed about the current study. In the current study, 35 monolingual Swedish infants (17 female) took part. All were full-term (gestational age week 38 or later; $M=40.51$ weeks, $S D=0.92)$. At time of testing their mean age was 9.38 months $(M=285.2$ days, $S D=$ 10.04 , range $268-309$ days). The parents of the participating infants reported that their infants were healthy and typically developing. These parents were highly educated ( $83 \%$ of mothers and $63 \%$ of fathers had a university degree). An additional 28 infants began the protocol but were not included in the final sample. Of the 28 infants, 15 failed to complete the protocol due to fussiness (i.e. actively trying to get away from watching the screen), and 13 infants did not meet inclusion criteria of the test protocol (i.e. saccade latencies identified for less than half of all trials in at least one block, see below). This rate of attrition is common in infant eye tracking studies that use parameters similar to those of the current experiment (e.g., Exp. 2, Kirkham et al., 2007; Exp. 2, Wang et al., 2012).

Adult comparison group. As a comparison group, 31 adults (18 female) with a mean age of 24 years ( $S D=3.18$, range $20-37$ years) working or studying at Linköping University, Sweden, completed testing. All were healthy and had normal or corrected to normal vision. A further 5 adults began the experiment, but their data could not be included due to technical failure $(n=3)$, experimenter error $(n=1)$, or failure of the system to detect the eyes during eye-tracking $(n=1)$.

\section{Ocular-motor serial reaction time task}



an eye tracking paradigm and the same protocol was run with infants and adults. For infants and adults, reaction times were measured by saccade latencies. tracker (Tobii AB, Stockholm, Sweden). The screen dimensions of the monitor were $1280 \mathrm{x}$ 1024 pixels. The firmware version of the T120 was 2.0.7. We used a 64-bit windows computer with operating system Windows 7 Enterprise (service pack 1). The processor was a 3.60 GHz Intel(R) CPU with $12 \mathrm{~GB}$ RAM. Tobii studio 3.4.8 was used to calibrate the eye tracking system to the participants' eyes and the experiment was run on MATLAB (MathWorks Inc, Massachusetts, USA) version 2016b. The custom script used for running the experiment utilized Psychtoolbox, version 3.0.13 (Brainard, 1997; Kleiner, Brainard, \& Pelli, 2007) and TobiiPro SDK (Tobii AB, Stockholm Sweden), version 1.1.0.21, for MATLAB. and cartoon characters (see Figure 1). They were presented with a size of $400 \times 400$ pixels, corresponding to 9.9 visual degrees for a viewing distance of about $60 \mathrm{~cm}$, the approximate distance between the participant's eyes and the screen. The three onscreen positions of the images (lower left corner, lower right corner, top center) were defined by an equidistant triangle with a distance of 14.9 visual degrees between the images' centers. This implementation of the ocular-motor SRT is based on gaze contingency. This means that trials were started based on gaze positions. Once a fixation on the target image was registered a new trial was triggered automatically by the system and a new image was displayed at a new spatial location. The procedure always started with an image that was presented at the center of the screen. After the participant had fixated on this image for at least 200 ms the image disappeared, and simultaneously the first trial of the SRT task 
started. In each trial, a new image appeared at the spatial location immediately after the previous image disappeared. The spatial location of the item (location A, B or C, see Figure 1) was determined either by the visual spatial sequence (during sequence block trials, details see below) or following a pseudorandom order (during random block trials, see below). Although the spatial location sequence repeated in the sequence trial block, different images were used on each trial. To ensure that the participant oriented to the screen, each trial onset was accompanied by one of 85 short unrelated infant friendly sounds that lasted less than a second. These sounds were related neither to the image nor the location. Each new trial began only after participants fixated for at least $200 \mathrm{~ms}$ on the area where the current image was being shown.

In this implementation, participants were presented with five blocks of 25 trials each. Blocks 1, 2, 3, and 5 were 'sequence blocks', while block 4 was a 'random block'. In each sequence block a 5 -item sequence was presented 5 times. The visuospatial sequence was A-B-C-A-B (following Vicari et al., 2003), with each letter corresponding to one of three spatial locations on screen. Infants were tested with one of two versions in order to control for possible directional biases in visual spatial sequence learning (see Bulf, de Hevia, Gariboldi, \& Macci Cassia, 2017, who found a directional bias for sequence learning in infancy). In the clockwise version, A corresponded to lower left corner, B to top center, and C to lower right corner for on screen position, with most transitions in the clockwise direction. In the counterclockwise version, A corresponded to top center, B to the lower left corner and $\mathrm{C}$ to lower right corner (see Figure 1). All trials followed directly after each other, with no indication of when the sequence or blocks ended or began. In the random block (block 4), a pseudo-random order of 25 visuospatial locations was used, which was C-B-A-CB-A-C-B-C-B-A-B-A-C-A-C-B-A-B-A-B-A-B-A-C for the clockwise version. And the reversed 
order was used in the counterclockwise version. It is important to note that reversals

(Vaquero, Jiménez, \& Lupiáñez 2006) were frequent in both the sequence and random blocks: 10 in the sequence and 12 in the random block. Please note reversal occurred when sequences were repeated (... A-B-C-A-B-A-B-C-A-B ... ).

If necessary, the experimenter could attract the infant's attention to the screen by activating an attention-getter. This changed the background color and replayed the infant friendly sound that was played on that trial's onset. The attention-getter was activated on average 5.11 times $(S D=6.52)$ per infant. On these trials, valid saccade latencies could still be obtained as the attention getter attracted the infant to the screen and the next trial would be trigged automatically after the infant fixated on the relevant area for $200 \mathrm{~ms}$ (see below for details). Further, the experimenter could manually trigger the next trial; this occurred on average in 0.23 trials $(S D=0.69)$ per infant. These trials could not yield valid saccade latencies and were automatically dropped from analyses.

On line data processing. While participants were being tested, gaze data was analyzed automatically in order to identify fixations on target images that would trigger the following trial contingent upon participant's gaze. At any one time during the experiment, the system would analyze the 26 most recent gaze data samples. Each gaze data sample consisted of $x$ and $y$ coordinates that define the position of the gaze on screen and these were collected with a frequency of $120 \mathrm{~Hz}$ (every $8.33 \mathrm{~ms}$ ). Thus, the 26 most recent gaze data samples assessed an infant's gaze behavior for at least the last $200 \mathrm{~ms}$. These data were "smoothed" by replacing each value by a mean using a "moving average" with a window of 5 data points. A fixation was then identified if during this time period no eye movement that was faster than the threshold of 35 degrees per second was detected. In this case, the position of the fixation was estimated by calculating the mean for all $\mathrm{x}$ and $\mathrm{y}$ coordinates 
during the last $200 \mathrm{~ms}$. If the position of the fixation matched the position of the current image, the next trial was trigged by displaying a new image at a new location and removing the current image. However, if no fixation was identified or the identified fixation was not on target, the system would analyze the 26 most recent data samples again. If a new data sample had been collected by the system in the interim, the data sample would be updated before the analysis was repeated. This ensured that the time lag of the system was no more than a few milliseconds and that the participant had fixated for at least $200 \mathrm{~ms}$ on the current image before the next trial was triggered.

\section{Procedure}

When an infant and parent arrived at the lab, the procedure was explained to the parent who then signed a consent form. Once the infant showed signs of comfort, such as smiles or positive vocalizations, the experimental procedure was started. The infant was seated in his or her parent's lap in front of the Tobii T120 monitor. A 36 sec infant friendly video clip was used to attract attention to the screen. While the infant watched the video, the distance from the monitor to the infant was adjusted to approximately $60 \mathrm{~cm}$, with the monitor centered in front of the infant's face.

Parents were instructed not to comment on what was shown on screen. They were also informed that it did not matter if the infant looked away for a short while, but if the infant did not attend to the monitor for a longer period, the parent could try to direct the infant's attention to the monitor by saying "look at that" in Swedish.

After the monitor's position was adjusted, the experimenter started the Tobii studio infant calibration procedure using five calibration points. A curtain that separated the experimenter from the parent and infant was closed before starting the calibration procedure. After successful calibration, several stimulus videos (total duration $244 \mathrm{~s}$ ) were 
shown. These were unrelated to the SRT task and part of a larger study. The SRT task started once the other videos were finished. Infants completed the SRT task on average in $104.3 \mathrm{sec}$ $(S D=22.5)$ with the longest duration of $169 \mathrm{sec}$.

Adults were tested using the same setup and stimuli as was used with infants. Since adults were recruited to participate in a Babylab study, they were informed that they were part of a comparison group in an experiment designed to test a newly adapted task for infants. After adult participants signed a consent form, they were asked to sit down in front of the Tobii T120 monitor and the calibration procedure was started. For adults, the SRT test started immediately after calibration. After they finished the SRT task, they were asked "Did you notice anything in particular during the test situation?" If the said yes, they were asked to expand. Participant could for instance refer to sounds, pictures or patterns. If the mentioned a pattern, they were asked to describe the pattern.

\section{Statistical analysis}

In the current study, the analyzed responses were saccade latencies. Saccade latencies were included only for trials in which a saccade from the location of the previous image to the location of the current image occurred between 0.1 and 2 seconds from trial onset. Criteria for identification of saccade latencies followed Kenward et al. (2017). Saccade latencies were extracted for raw eye gaze data by a custom-made script using MATLAB 2016b. Gaze data was processed by first removing all invalid data points, as defined by Tobii studio. If possible, gaze data position was based on an average for both eyes' gaze positions. If data for only one eye was available these data were used. As with the on-line analysis, data were smoothed by applying a moving average over five samples to $\mathrm{x}$ and $\mathrm{y}$ coordinates separately, then eye movement velocity was calculated and saccades were defined as periods of eye movements above 35 degrees per second. In order to avoid data 
misclassification, response saccades are classified as those saccades whose origin matched the position of the previous image and whose destination matched the position of the current image. All periods of eye movement above 35 degrees per second occurring between 100 ms and either 2000 ms or the beginning of the next trial (which ever occurred first) were analyzed for origin and destination. If origin and destination matched, the response saccade was identified and latency calculated from trial onset to the beginning of the saccade.

Participants were included in the analyses if they completed all five blocks. Due to infants being more easily distracted than adults, an additional inclusion criterion for infants was used. For infants, saccade latencies had to be identified for at least half of all trials per block (for a minimum of 13 of the 25 trials per block). Data for 35 infants meet this criterion but 13 additionally tested infants were excluded for not meeting this criterion (see Participants). This ensured that the mean for a given block is based on at least 13 valid saccade latencies from that block and that infants were thus following the procedure as intended.

First, to test whether infants and adults exhibited sequence learning, we analyzed performance on the critical phase sequence block 3 , random block and final sequence block. Following Lum, Conti-Ramsden, Page, and Ullman (2012), each individual's saccade latencies were transformed to z-scores. Transformation to a z-score for a given trial was calculated based on an individual's median and SD for all valid trials in these three blocks. The transformation ensures that all participants' shortest and longest saccade latencies had similar values. Because the data were positively skewed, the median rather than the mean was more representative of the central tendency for the transformation. For this reason, the median was used as an anchor when transforming each individual's data to z-scores. The 
mean of the transformed data for each block was then calculated based on z-values for all valid trials for a given block per participant. This resulted in 3 mean z-values per participant for saccade latencies. Changes between blocks were compared by a mixed-design analysis of variance (ANOVA) with repeated measures across blocks and factors such as age group (infant or adult) or gender as between group measures.

Further, the learning phase was also analyzed based on z-scores. Here saccade latencies were transformed using the median and SD for all valid saccade latencies in sequence block 1, 2, and 3 to calculate z-scores for each valid trial. As above, for each block (block 1 to 3 ) a z-score mean was then calculated, which was used as the dependent variable in the repeated measures ANOVA. Mauchly's test of sphericity was not significant for any of the presented analyses. For post-hoc comparisons differences between groups, Bonferroni corrections were used throughout. Statistical analyses were performed in MATLAB ${ }^{\circledR} 2016 b$. Partial eta square is reported following the definition of Richardson (2011). Files for gaze data and the $M A T L A B^{\circledR}$ script that extracts the saccade latencies and performs the analyses are published and available for download (Koch et al, under review).

\section{Results}

To illustrate the raw data pattern of results, descriptive statistics for raw saccade latencies in milliseconds can be found in Table 1. As Table 1 shows, saccade latencies of infants are nearly twice that of adults and also the variability in saccade latencies of infants is nearly twice that of adults. All the subsequent analysis are based on z-transformed values (see method section for details). Due to the debate about whether data should be normalized (Janacsek et al., 2012; Lukács \& Kemény, 2015; Zwart et al., 2017), all analyses were performed with raw scores, as well. These results can be found in the supplemental material, showing that the findings and conclusion are the same, irrespective of whether 
data were normalized. First, the critical phase (sequence block 3, random block, and final sequence block) of the ocular-motor SRT is examined. Next, we conducted an analysis to examine the learning phase (sequence block 1 to 3 ).

\section{Preliminary analyses of the critical phase}

We first assessed whether gender or sequence order was associated with task performance. Gender did not enter into any significant main effects, $F(1,62)=0.18, p=.67$, $\eta_{\mathrm{p}}{ }^{2}<.01$, or any 2 -way or 3-way interaction terms (all ps $>$.1). For infants, order (clockwise v. counterclockwise) was tested but this factor did not enter any main effects, $F(1,31)=1.65, p$ $=.21, \eta_{\mathrm{p}}{ }^{2}=.05$, nor any 2-way or 3-way interactions (all $p s>.1$ ). The factors gender and order were not considered in any subsequent analyses.

\section{Performance in the critical phase}

A mixed-design ANOVA was run with repeated measures across blocks ( 3 to 5 ) and between group factor age (infant vs adult). This analysis indicated that there was a main effect of block, $F(2,128)=5.71, p<.01, \eta_{p}^{2}=.08$, a main effect of age, $F(1,64)=5.39, p=$ $.02, \eta_{p}^{2}=.08$, but no interaction between block and age, $F(2,128)=0.37, p=.69, \eta_{p}^{2}<.01$. For both infants and adults there was a significantly longer mean reaction time in the random block compared to both the immediately preceding, $p=.02$, and immediately following recurring sequence blocks, $p<.01$.

\section{Learning Phase}

Further, it is of interest to analyze how saccade latencies changed during the learning phase, this concerns the sequence blocks 1 to 3. Again, a mixed-design ANOVA was run with repeated measures across blocks (here sequence blocks 1 to 3 ) and between group factor age (infant vs adult). This analysis yielded an interaction between age and block, $F(2$, $128)=8.65, p<.001, \eta_{p}^{2}=.12$, as well as main effect of age, $F(1,64)=8.64, p<.01, \eta_{p}^{2}=.12$, 
and block, $F(2,128)=18.76, p<.001, \eta_{p}{ }^{2}=.23$. For infants, differences in mean reaction time were not significant between block 1 and $2(p=.15)$, but decreased significantly from block 2 to $3(p=.02)$. For adults, differences were not significant between blocks (both $p^{\prime} s>$ .10). It is important to note that saccade latency did not significantly increase during the learning phase for either infants or adults, as such an increase during the learning phase might indicate that the task was not working as intended (e.g. due to fatigue of participants). Thus, an increase in saccade latency was only observed for the random sequence during the critical phase, see above.

\section{Adult explicit sequence knowledge}

Three participants reported that they noticed a sequence that was three items long $(A-B-C)$ and one participant reported a sequence that was four items long (A-B-A-C), but no one reported a five-item or longer sequence and thus no one identified a whole correct sequence. Eight participants said they were looking for a pattern but did not find one, seven participants commented that the images and sounds used were infant-friendly or funny, and six participants commented that images changed very quickly. Two participants said they were trying to match the sounds to the images but did not find a pattern there.

\section{Discussion}

Here, we tested a novel infant-friendly ocular-motor version of the SRT task adapted for infants utilizing saccade latencies as the main measure. Participants were presented with three sequence blocks, followed by a random block, and a final sequence block. Performance on the ocular-motor SRT task indicates sequence learning when mean reaction time on the random block is significantly slower compared to both the preceding and following recurring sequences blocks. This pattern was significant for all participants, infants and adults alike. For the learning phase, no significant deceleration in reaction times 
was observed, neither for adults nor for infants. This indicates that a deceleration in reaction time was unique to the random block. For infants, faster saccade latencies were apparent for block 3 compared to block 2 . This decrease in saccade latencies is in line with previous research on sequence learning showing better performance across the initial blocks (Magallón et al., 2016). The change in saccade latencies for infants may reflect general task learning effects. However, as deceleration in response time was only observed for the random block, the finding here shows specific sequence learning for infants and adults.

As can be expected based on previous research (Kenward et al., 2017), adults' overall saccade latencies were nearly twice as fast as those of infants and the infants showed a much higher variability in latencies compared to adults (see Table 1). Despite accounting for differences in absolute reaction time by normalizing the data, specific sequence learning effects were found in both infants and adults. As mentioned in the introduction, whether the data was normalized or not influenced the interpretation of developmental trends in some studies (Janacsek et al., 2012; Lukács \& Kemény, 2015; Zwart et al., 2017) and for this reason we chose the more stringent normalization approach in our analysis. Importantly, in the current study, the pattern of results did not differ between normalized data (see results section) and raw data (see Supplemental material); infants and adults showed similar changes in performance in the final three blocks. The mean reaction times of both infants and adults was slower in the random block compared to the preceding sequence block as well as the subsequent recurring sequence block. This suggests that participants in both age groups were able to use their knowledge of the sequence to restore their speed of response in the final sequence block. These findings suggest that developmental changes can be tracked from infancy to adulthood and using the same measure. Whether development of 
procedural memory is best described as developmentally invariant or entails developmental changes in performance is beyond the scope of the current study.

The development of an infant SRT task provides a number of possibilities for future research avenues to test theoretical models of procedural learning. Specifically, researchers have suggested (Kóbor et al., 2018, Simor et al., 2019) that procedural memory consists of two subprocesses, namely sequence learning and statistical learning. Whereas sequence learning refers to the ability to learn the serial order of longer sets of items (5-12) (Simor et al., 2019), statistical learning (Saffran \& Kirkham, 2018) refers to the ability to learn frequency or probability relations between items. Nemeth et al. (2013) suggested that there are different developmental trajectories for these two subprocesses of procedural memory from age 11 years and onwards. Nemeth and colleagues argue that although statistical learning decreases, higher-order sequence learning increases in efficiency from 11 years onward. Amso and Davidow (2012) suggested that there is developmental invariance from infancy in statistical learning, suggested by the same pattern of responses across age groups. But until now there has not been an SRT task suitable for use with infants in order to directly compare performance on statistical learning and sequence learning. The results presented here provide evidence of specific sequence learning effects (Robertson, 2007) in infants and adults. By assessing sequence learning (using the ocular-motor SRT task) and statistical learning (using e.g. Amso \& Davidow's task) in various age groups, future research could document and compare the developmental trajectory of both subprocesses of procedural memory.

Methodological modifications of the ocular-motor SRT task need further discussion. The visuospatial sequence used in the current study is shorter ( 5 items) than sequences that 
are usually presented to adult participants (10 items) (Nissen \& Bullemer, 1987, Robertsson,

477 2007). And three instead of four locations have been used. Despite these simplifications, of the adult participants, only four reported noticing any visuospatial sequence, and none of them identified the correct one. This may have been partly due to the fact that new images were presented on each trial, which is a deviation from previous SRT tasks for which dots or asterisks are used as stimuli. The images were primarily used to motivate attention for the infants but perhaps an unintended consequence was that adults were not aware of the regularity in the spatiotemporal locations of the sequence. That is, one effect could be that adults' attention focused more on the content of the images, making it harder to detect the visual spatial sequence. Despite the added complexity due to novel images on each trial, we speculate that this does not change the procedural aspect of the task but assume that the visual attractiveness of the images kept infants engaged with the task. All participant were given about $200 \mathrm{~ms}$ to attend to each image and thereafter the image automatically disappeared and the next trial was triggered. This reduced individual variation for how much visual attention a participant devoted to images and also controlled for visual attention between infants and adults. We have not examined the effect of stimulus material, but overall saccade latencies do not seem to be slower for 9-month-olds or adults in the present study (compare Kenward et al, 2017). Further, new images appeared simultaneously with the offset of the old image without any gap or delay between trials, which is contrary to common SRT tasks, that use a time delay of between 200 and 500 ms (Robertson, 2007). So participants could not "linger" on an image before responding as it disappeared after $200 \mathrm{~ms}$ of visual attention, just as the next image appeared. We think that the combination of gaze contingent trial onset and lack of delays between images made it difficult to detect and verbally describe this simplified sequence. More sophisticated screening methods for 
500

501

502

503

504

505

506

507

508

509

510

511

512

513

514

515

522

detecting awareness about the sequence (e.g. Stark-Inbar et al., 2017) would better describe to what degree adults are aware of the target sequence. Future research should cross-

validate the current method with a more traditional SRT method with adults to confirm that similar patterns of behavior emerge. Given the fact that the findings of this study point to implicit sequence learning by adults, it seems likely that the infants also learned the task implicitly. Thus, we propose that the newly adapted ocular-motor SRT task introduced in the present study provides a measure of procedural memory that can be used from infancy to adulthood.

\section{Limitations}

In total 63 infants started the test in the current study. Of these, 15 did not finish it due to fussiness, and a further 13 infants were excluded due to not passing the inclusion criterion, which was that more than half of the trials per block had to provide valid saccade latencies. Lack of valid saccade latencies could be due to improperly tracked eye gaze even though infants were looking at the screen. In order to improve the eye gaze data, more sophisticated gaze data imputation techniques could be used, which might identify more valid saccade latencies. Another reason for missing saccade latencies could be that infants did not saccade towards the upcoming image but looked away from the screen after a new trial was triggered. In these cases, infants disengaging from the task which would, if it happened too often jeopardize the validity of this task as a procedural task. Triggering trials without attending to the next trial would not lead to procedural learning. This was the reason for implementing the inclusion criterion for infants. Attrition is common in infancy studies and attrition in the current study is not different to what might be expected when studying infant cognition (Kirkham et al., 2007; Rovee-Collier \& Barr, 2002; Wang et al., 
2012). Our 9-month-old participants had been watching 4 minutes of television before starting the SRT procedure. Had we started with the SRT procedure earlier, fewer infants might have been lost due to fussiness. Further, inclusion criteria need to be balanced between ensuring that infants actually perform the intended task and including as many infants as possible (Oakes, 2010). Future studies, could examine improvements of inclusion criteria for infants.

For adults, the increase in saccade latencies in the random condition is much lower than Vakil et al. (2017) observed, in a sequence learning study. Several differences might contribute to our finding. As mentioned above we used meaningful images that were novel for each trial, but we also used three instead of four positions. The main difference when it comes to motor behavior is that that Vakil et al. changed the sequence after participants viewed 648 trials, as opposed to 75 trials in the current study. As procedural memory depends on practice, a longer learning phase is likely to lead to a stronger disruption by the random sequence. The much lower number of trials in our study compared to other prior studies could also be a reason for adults not noticing the correct visuospatial sequence. This could be verified empirically.

Vaquero et al. (2006) pointed to the problems of reversals during sequence learning. Reversals are found when the target appears in the same area in the first and third trial of three consecutive trials. Vaquero et al argue that reversals occur more often in random sequences than in structured sequences. With only three positions to choose from, reversals were rather common in the current study in both conditions (10 in sequence blocks and 12 in the random block). Vaquero et al. recommend using a different structured sequence as the control comparison. In the current experiment, we used two versions, a 
clockwise and a counterclockwise version, during sequence learning and compared these to when the sequence was random. It might be possible to use the counterclockwise version as the control sequence when infants learned the clockwise version (and vice versa). We have not tested this. Another methodological change could be using four positions, instead of three. With more positions to choose from this could also reduce the problem with reversals, but whether infants can learn the structure in a sequence that is based on four locations remains to be tested. Establishing the SRT protocol for infants means that it will be possible to test these variations.

$$
\text { Recently, issues about reliability of SRT and related tasks have been discussed }
$$
(Conway, Arciuli, Lum, \& Ullman, 2019; West, Vadillo, Shanks, \& Hulme, 2018). A limitation of the ocular-motor SRT presented here, is the lack of test-retest data. Infants have only been tested once. Infants could be tested several times in order to assess consistency in procedural learning.

\section{Future directions}

Theoretically it is important to develop several memory measures that can track the development of different memory systems over the life course. The development of such measures would allow researchers to track the emergence of abilities that depend on specific memory systems, but also to examine whether each system emerges independently of each other or if they are dependent on each other. With respect to procedural memory, the adapted ocular-motor SRT task could be used to track encoding, consolidation and recall processes. Of particular interest would be the issue of memory consolidation during sleep (Fisher, Drosopoulos, Tsen \& Born, 2006). 
memory in infants. By using the same SRT task from infancy through childhood and into adulthood the development trajectory of sequence learning can be examined. A possible developmental invariance or a decline in saccade latencies might be possible outcomes. It is not clear if the adapted SRT task works for the elderly population and this is an interesting future examination.

Whether or not infants can also learn sequences that reflect second order dependencies (and not just first order dependencies as used here) remains to be seen. Firstand second-order sequence learning might rely on distinct neural networks in adults (Clark et al., 2019; Lum et al., 2018). If infants are not able to learn second-order dependencies, this might be an aspect of the procedural memory system that develops with age. Future research could investigate whether this possible change is due to better integration of the procedural memory system with other systems or changes in the procedural memory system itself. It may be possible to develop an alternating SRT variant of this task.

The ocular-motor SRT protocol outlined in this study takes less than three minutes to complete, which makes it usable for infant research. This also means that the task can be integrated in a protocol that includes other cognitive measures, allowing procedural memory to be compared to other cognitive abilities and their development. In adults, it has been demonstrated that implicit sequence learning, which the SRT task entails, relies on a network of brain structures that is rooted in the basal ganglia, in particular portions of the striatum (Clark et al., 2014; Hardwick, Rottschy, Miall, \& Eickhoff, 2013). Neuroimaging studies have demonstrated dissociations between procedural and declarative memory systems in adults (Squire, 2009). The current task provides a promising tool to examine the developmental origin of the dissociation between memory systems. 


\section{Conclusion}

593 The novel infant friendly ocular-motor adaptation of the SRT task introduced here

594 enables rapid assessment of implicit sequence learning and is a promising procedure for

595 measuring procedural memory in infants. The results indicate that infants show procedural 596 memory similar to that of adults.

597 


\section{References}

Adi-Japha, E., Badir, R., Dorfberger, S., \& Karni, A. (2014). A matter of time: Rapid motor memory stabilization in childhood. Developmental Science, 17, 424-433. https://doi.org/10.1111/desc.12132

Amso, D., \& Davidow, J. (2012). The development of implicit learning from infancy to adulthood: item frequencies, relations, and cognitive flexibility. Developmental Psychobiology, 54, 664-673.

Braddick, O., \& Atkinson, J. (2011). Development of human visual function. Vision Research, 51(13), 1588-1609. https://doi.org/10.1016/j.visres.2011.02.018

Brainard, D. H. (1997). The Psychophysics Toolbox, Spatial Vision, 10, 433-436

Bulf, H., de Hevia, M. D., Gariboldi, V., \& Macci Cassia, V. (2017). Infants learn better from left to right: A directional bias in infants' sequence learning. Scientific Reports, 7, 2437. https://doi.org/10.1038/s41598-017-02466-w

Canfield, R. L., \& Haith, M. M. (1991). Young infants' visual expectations for symmetric and asymmetric stimulus sequences. Developmental Psychology, 27, 198-208. https://doi.org/10.1063/1.1136086

Canfield, R. L., Smith, E. G., Brezsnynak, M. P., \& Snow, K. L. (1997). Information processing through the first year of life: A longitudinal study using the visual expectation paradigm. Monographs of the Society for Research in Child Development, 62, (2, Serial No. 250).

Clark, G. M., Barham, M. P., Ware, A. T., Plumridge, J., O'Sullivan, B., Lyons, K., ... \& Enticott, P. G. (2019). Dissociable implicit sequence learning mechanisms revealed by continuous theta-burst stimulation. Behavioral Neuroscience.

Clark, G. M., Lum, J. A. G., \& Ullman, M. T. (2014). A meta-analysis and meta-regression of serial reaction time task performance in Parkinson's disease. Neuropsychology, 28(6), 
945-958. https://doi.org/10.1037/neu0000121

Conway, C. M., Arciuli, J., Lum, J. A., \& Ullman, M. T. (2019). Seeing problems that may not exist: A reply to West et al.'s (2018) questioning of the procedural deficit hypothesis. Developmental Science, e12814.

Eichenbaum, H. (2010). Memory systems. WIREs Cognitive Science, 1, 478-490. DOI: $10.1002 /$ wcs.49

Esser, S., \& Haider, H. (2017). The emergence of explicit knowledge in a serial reaction time task: The role of experienced fluency and strength of representation. Frontiers in Psychology, 8, 502.

Fischer, S., Drosopoulos, S., Tsen, J., \& Born, J. (2006). Implicit learning - explicit knowing: A role for sleep in memory system interaction. Journal of Cognitive Neuroscience, 18, 311319. http://dx.doi.org/10.1162/089892906775990598

Haith, M. M., Hazan, C., \& Goodman, G. S. (1988). Expectation and anticipation of dynamic visual events by 3.5-month-old babies. Child Development, 59, 467. https://doi.org/10.2307/1130325

Hardwick, R. M., Rottschy, C., Miall, R. C., \& Eickhoff, S. B. (2013). A quantitative metaanalysis and review of motor learning in the human brain. Neurolmage, 67, 283-297. https://doi.org/10.1016/j.neuroimage.2012.11.020

Howard, J. H., \& Howard, D. V. (1997). Age differences in implicit learning of higher order dependencies in serial patterns. Psychology and Aging, 12, 634.

Howard, J. H., \& Howard, D. V. (2013). Aging mind and brain: is implicit learning spared in healthy aging?. Frontiers in Psychology, 4, https://doi.org/817. 10.3389/fpsyg.2013.00817

Howard, J. H., Howard, D. V., Dennis, N. A., \& Kelly, A. J. (2008). Implicit learning of predictive 
relationships in three-element visual sequences by young and old adults. Journal of Experimental Psychology: Learning Memory and Cognition, 34, 1139-1157. https://doi.org/10.1037/a0012797

Jabès, A., \& Nelson, C. A. (2015). 20 years after "the ontogeny of human memory: A cognitive neuroscience perspective," where are we? International Journal of Behavioral Development, 39, 293-303. https://doi.org/10.1177/0165025415575766

Janacsek, K., Fiser, J., \& Nemeth, D. (2012). The best time to acquire new skills: Age-related differences in implicit sequence learning across the human lifespan. Developmental Science, 15, 496-505. https://doi.org/10.1111/j.1467-7687.2012.01150.x

Jones, E. J. H., \& Herbert, J. S. (2006). Exploring memory in infancy: deferred imitation and the development of declarative memory. Infant and Child Development, 15, 195-205.

Karatekin, C., Marcus, D. J., \& White, T. (2007). Oculomotor and manual indexes of incidental and intentional spatial sequence learning during middle childhood and adolescence. Journal of Experimental Child Psychology, 96, 107-130.

Kenward, B., Koch, F.-S., Forssman, L., Brehm, J., Tidemann, I., Sundqvist, A., ... Gredebäck, G. (2017). Saccadic reaction times in infants and adults: Spatiotemporal factors, gender, and interlaboratory variation. Developmental Psychology, 53, 1750-1764. https://doi.org/10.1037/dev0000338

Kirkham, N. Z., Slemmer, J. A., Richardson, D. C., \& Johnson, S. P. (2007). Location, location, location: Development of spatiotemporal sequence learning in infancy. Child Development, 78, 1559-1571. https://doi.org/10.1111/j.1467-8624.2007.01083.x Kleiner, M., Brainard, D., \& Pelli, D. (2007). What's new in Psychtoolbox-3?, Perception, 36, ECVP Abstract Supplement.

Kóbor, A., Takács, Á., Kardos, Z., Janacsek, K., Horváth, K., Csépe, V., \& Nemeth, D. (2018). 
ERPs differentiate the sensitivity to statistical probabilities and the learning of sequential structures during procedural learning. Biological psychology, 135, 180-193. Koch, F.-S., Sundqvist, A., Birberg Thornberg, U., Ullman, M.T., Barr, R., Rudner, M., \& Heimann, M. (under review). Data and analysis script for infant and adult eye movement in an adapted ocular-motor serial reaction time task assessing procedural memory. Data in Brief.

Lieberman, M. D. (2000). Intuition: a social cognitive neuroscience approach. Psychological Bulletin, 126, 109.

Lukács, Á., \& Kemény, F. (2015). Development of different forms of skill learning throughout the lifespan. Cognitive Science, 39, 383-404.

Lum, J., Conti-Ramsden, G., Page, D., \& Ullman, M. T. (2012). Working , declarative and procedural memory in specific language impairment. Cortex, 48, 1138-1154. https://doi.org/10.1016/j.cortex.2011.06.001

Lum, J., Kidd, E., Davis, S., \& Conti-Ramsden, G. (2010). Longitudinal study of declarative and procedural memory in primary school-aged children. Australian Journal of Psychology, $62,139-148$.

Lum, J., Mills, A., Plumridge, J. M., Sloan, N. P., Clark, G. M., Hedenius, M., \& Enticott, P. G. (2018). Transcranial direct current stimulation enhances retention of a second (but not first) order conditional visuo-motor sequence. Brain and Cognition, 127, 34-41.

Lum, J. \& Ullman, M.T., \& Conti-Ramsden, G. (2013) Procedural learning is impaired in dyslexia: Evidence from a meta-analysis of serial reaction time studies. Research in Developmental Disabilities 34, 3460-3476.

http://dx.doi.org/10.1016/j.ridd.2013.07.017

Magallón, S., Narbona, J., \& Crespo-Eguílaz, N. (2016). Acquisition of motor and cognitive 
skills through repetition in typically developing children. PLOS ONE, 11, e0158684. https://doi.org/10.1371/journal.pone.0158684

Manns, J. R., Stark, C. E., \& Squire, L. R. (2000). The visual paired-comparison task as a measure of declarative memory. Proceedings of the National Academy of Sciences of the United States of America, 97, 12375-12379. doi:10.1073/pnas.220398097

Marcus, D. J., Karatekin, C., \& Markiewicz, S. (2006). Oculomotor evidence of sequence learning on the serial reaction time task. Memory and Cognition, 34, 420-432. https://doi.org/10.3758/BF03193419

Mullally, S. L., \& Maguire, E. A. (2014). Learning to remember: The early ontogeny of episodic memory. Developmental Cognitive Neuroscience, 9, 12-29. https://doi.org/10.1016/j.den.2013.12.006

Nemeth, D., Janacsek, K., \& Fiser, J. (2013). Age-dependent and coordinated shift in performance between implicit and explicit skill learning. Frontiers in Computational Neuroscience, 7, 147.

Nissen, M. J., \& Bullemer, P. (1987). Attention requirements of learning evidence from performance measures. Cognitive Psychology, 19, 1-32.

Oakes, L. M. (2010) Using Habituation of Looking Time to Assess Mental Processes in Infancy, Journal of Cognition and Development, 11, 255-268, DOI: 10.1080/15248371003699977

Reznick, J. S., Chawarska, K., \& Betts, S. (2000). The development of visual expectations in the first year. Child Development, 71, 1191-1204. https://doi.org/10.1111/14678624.00223

Richardson, J. T. E. (2011). Eta squared and partial eta squared as measures of effect size in educational research. Educational Research Review, 6, 135-147. https://doi.org/10.1016/j.edurev.2010.12.001 
Richmond, J., \& Nelson, C. A. (2007). Accounting for change in declarative memory: A cognitive neuroscience perspective. Developmental Review, 27, 349-373. https://doi.org/10.1016/j.dr.2007.04.002

Richmond, J., Sowerby, P., Colombo, M., \& Hayne, H. (2004). The effect of familiarization time, retention interval, and context change on adults' performance in the visual paired-comparison task. Developmental Psychobiology, 44, 146-155.

Robertson, E. M. (2007). The serial reaction time task: implicit motor skill learning? Journal of Neuroscience, 27, 10073-5. DOI:10.1523/JNEUROSCI.2747-07.2007

Rovee-Collier, C. \& Barr, R. (2002). Infant cognition. In H. Pashler (Series Ed.; J. Wixted, Vol. Ed.), Stevens' handbook of experimental psychology (3rd ed.). Vol. 4: Methodology (pp. 693-791). New York: Wiley

Saffran, J. R., \& Kirkham, N. Z. (2018). Infant statistical learning. Annual Review of Psychology, 69, 181-203.

Schacter, D. L., Wagner, A. D., \& Buckner, R. L. (2000). Memory systems of 1999. In E. Tulving \& F. I. M. Craik (Eds.), The Oxford Handbook of Memory, 627-643. New York, NY, US: Oxford University Press.

Scerif, G., Karmiloff-Smith, A., Campos, R., Elsabbagh, M., Driver, J., \& Cornish, K. (2005). To look or not to look? Typical and atypical development of oculomotor control. Journal of Cognitive Neuroscience, 17, 591-604. https://doi.org/10.1162/0898929053467523

Simor, P., Zavecz, Z., Horvath, K., Elteto, N., Török, C., Pesthy, O., ... \& Nemeth, D. (2019). Deconstructing procedural memory: Different learning trajectories and consolidation of sequence and statistical learning. Frontiers in Psychology, 9, 2708.

Song, S., Howard, J. H., \& Howard, D. V. (2007). Implicit probabilistic sequence learning is independent of explicit awareness. Learning \& Memory, 14, 167-76. 
doi:10.1101/Im.437407

Squire, L. R. (2004). Memory systems of the brain: A brief history and current perspective. Neurobiology of Learning and Memory, 82, 171-177. https://doi.org/10.1016/j.nlm.2004.06.005

Squire, L. R. (2009). Memory and brain systems: 1969-2009. The Journal of Neuroscience, 29, 12711-6. DOI:10.1523/JNEUROSCI.3575-09.2009

Stark-Inbar, A., Raza, M., Taylor, J. A., \& Ivry, R. B. (2016). Individual differences in implicit motor learning: task specificity in sensorimotor adaptation and sequence learning. Journal of europhysiology, 117, 412-428.

Ullman, M. T. (2016). The declarative/procedural model: A neurobiological model of language learning, knowledge and use. In G. Hickok \& S. A. Small (Eds.), The Neurobiology of Language (pp. 953-68). Elsevier.

Vakil, E., Bloch, A., \& Cohen, H. (2017). Anticipation measures of sequence learning: manual versus oculomotor versions of the serial reaction time task. Quarterly Journal of Experimental Psychology, 70(3), 579-589.

https://doi.org/10.1080/17470218.2016.1172095

Vaquero, J. M., Jiménez, L., \& Lupiáñez, J. (2006). The problem of reversals in assessing implicit sequence learning with serial reaction time tasks. Experimental Brain Research, 175, 97-109.

Vicari, S., Marotta, L., Menghini, D., Molinari, M., \& Petrosini, L. (2003). Implicit learning deficit in children with developmental dyslexia, Neuropsychologia, 41, 108-114, https://doi.org/10.1016/S0028-3932(02)00082-9

Wang, Q., Bolhuis, J., Rothkopf, C.A., Kolling, T., Knopf, M., \& Triesch, J. (2012) Infants in Control: Rapid Anticipation of Action Outcomes in a Gaze-Contingent Paradigm. PLoS 
ONE, 7, e30884. https://doi.org/10.1371/journal.pone.0030884

West, G., Vadillo, M. A., Shanks, D. R., \& Hulme, C. (2018). The procedural learning deficit hypothesis of language learning disorders: we see some problems. Developmental Science, 21, e12552.

Yordanova, J., Kirov, R., \& Kolev, V. (2015). Increased performance variability as a marker of implicit/explicit interactions in knowledge awareness. Frontiers in Psychology, 6, 1957.

Zwart, F. S., Vissers, C. T. W. M., Kessels, R. P. C., \& Maes, J. H. R. (2017). Procedural learning across the lifespan: A systematic review with implications for atypical development. Journal of Neuropsychology. https://doi.org/10.1111/jnp.12139 


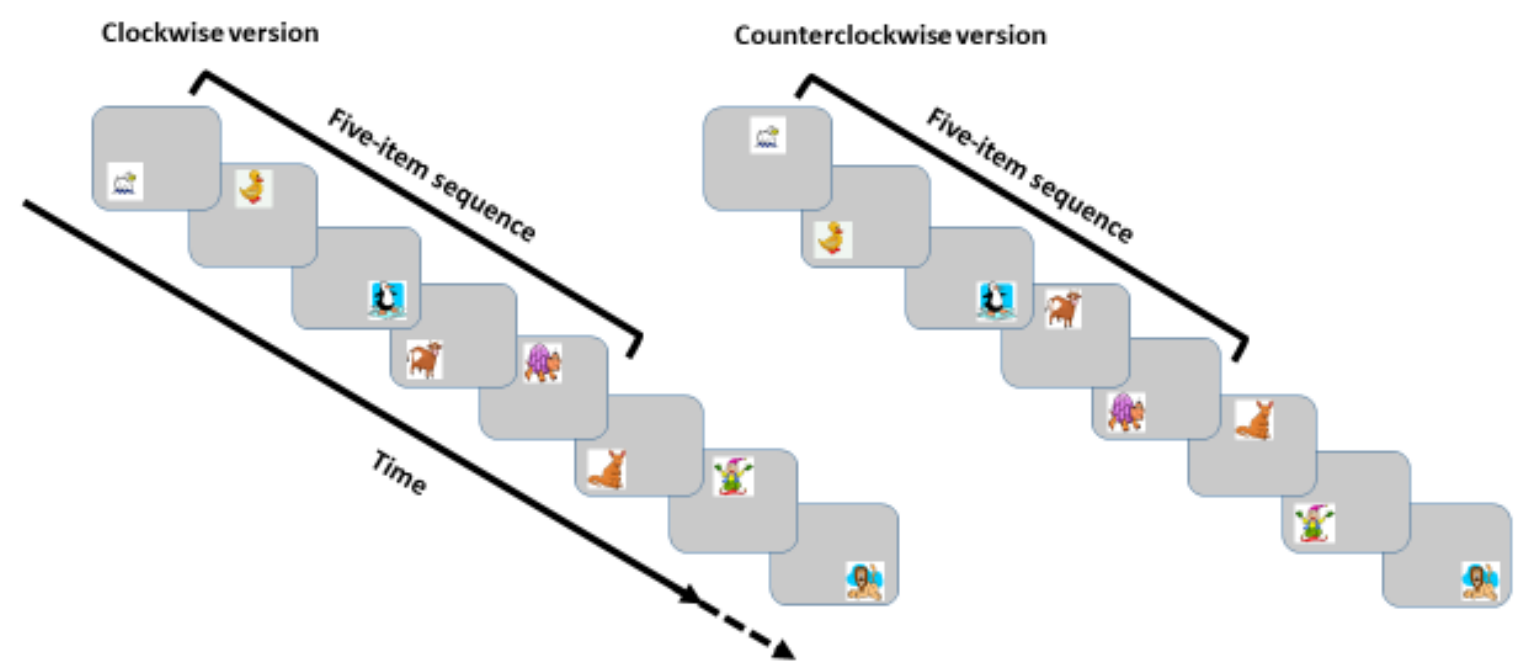

Captation Figure 1

Figure 1. Infant friendly ocular-motor serial reaction time task. Images are shown in one of three different locations ( $A, B$, and $C$ ), one at a time. After the infant fixated on an image for at least 200 ms, it disappeared and the next image immediately appeared at a new spatial location. Order of spatial locations is determined by a 5-item sequence (A-B-C-A-B) that was repeated five times during each sequence block. Each infant saw either a clockwise version, where positions $A, B$, and C corresponded to lower left corner, top center, and lower right corner, respectively or a counterclockwise version, where positions $A, B$, and C corresponded to top center, lower left corner, and lower right corner, respectively. 
2 Table 1 for manuscript entitled: Procedural memory in infancy: Evidence from implicit sequence learning in an eye-tracking paradigm

Table 1: Mean and SD for saccade latencies in milliseconds for infants and adults per block.

\begin{tabular}{|c|c|c|c|c|c|c|c|c|c|c|c|}
\hline \multirow[b]{2}{*}{ Group } & \multirow[b]{2}{*}{$\mathrm{N}$} & \multicolumn{2}{|c|}{$\begin{array}{c}\text { Block } 1 \\
\text { Sequence }\end{array}$} & \multicolumn{2}{|c|}{$\begin{array}{c}\text { Block } 2 \\
\text { Sequence }\end{array}$} & \multicolumn{2}{|c|}{$\begin{array}{c}\text { Block } 3 \\
\text { Sequence }\end{array}$} & \multicolumn{2}{|c|}{$\begin{array}{l}\text { Block } 4 \\
\text { Random }\end{array}$} & \multicolumn{2}{|c|}{$\begin{array}{c}\text { Block } 5 \\
\text { Sequence }\end{array}$} \\
\hline & & Mean & SD & Mean & SD & Mean & SD & Mean & SD & Mean & SD \\
\hline Infants & 35 & 347.7 & 50.5 & 334.7 & 44.8 & 319.9 & 46.0 & 328.3 & 52.0 & 318.5 & 48.9 \\
\hline Adults & 31 & 186.6 & 22.8 & 183.3 & 25.8 & 180.9 & 16.8 & 190.5 & 26.8 & 183.5 & 18.3 \\
\hline
\end{tabular}

6 


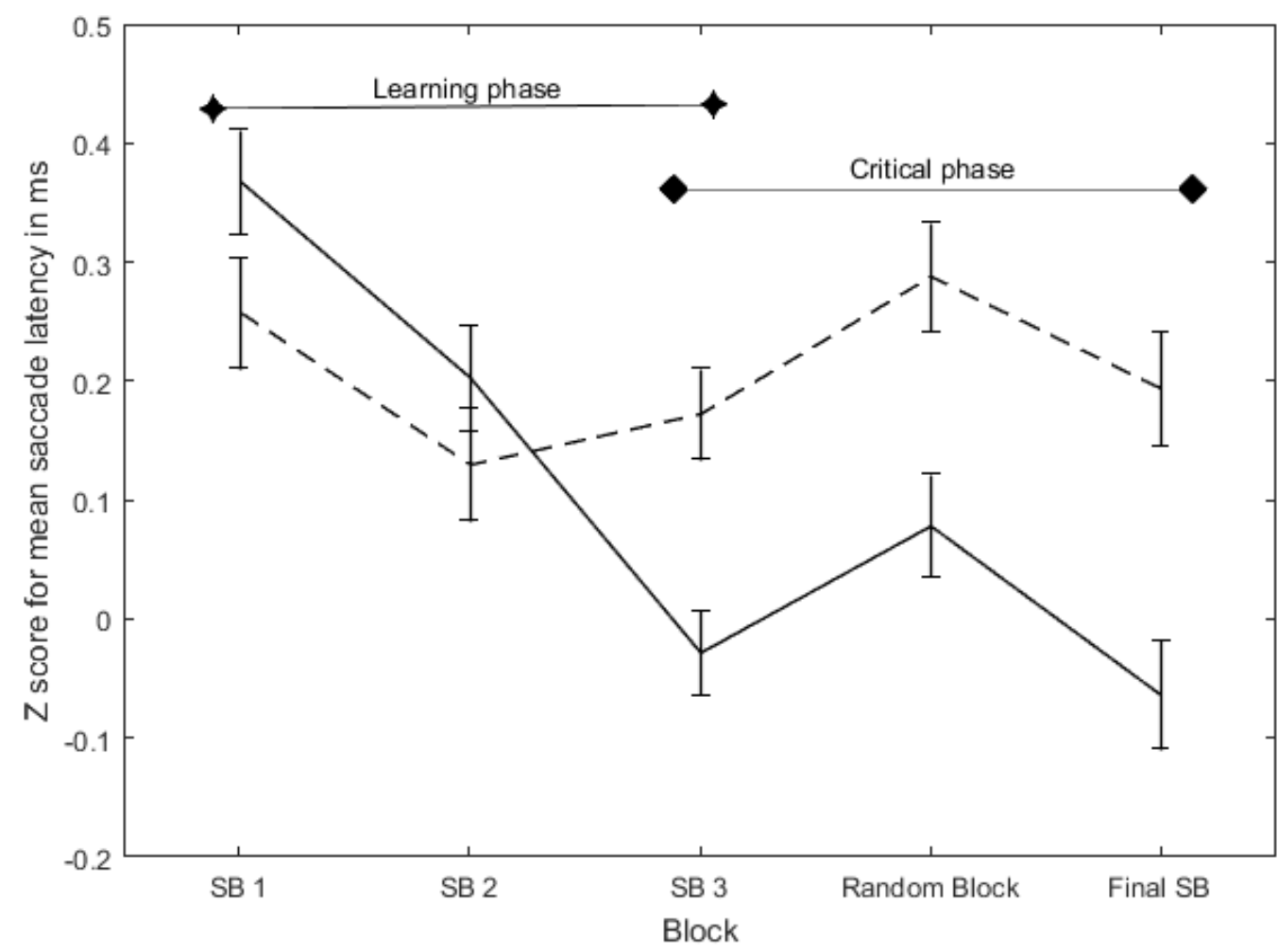

8

9

10

11

12

13

14

15

16

17 2

\section{Captation Figure 2}

3 Figure 2. Infants' (solid line) and adults' (dashed line) means for z-scores per block. SB:

4 Sequence block. The median is used as a reference for the z-transformation. This is why 5 most values are higher than 0. 